\title{
Oral Health Disparities in 6-9 years old Pakistani School Going Children
}

\author{
Sarah Hamid \\ Riphah International University \\ Islamabad, Pakistan \\ Email: ssarahhamid [AT] hotmail.com
}

\begin{abstract}
----
Introduction: Pakistan, a developing Asian country, faces many challenges in delivering oral health needs. The main problem of Pakistani population especially in rural area, where majority of population resides, is lack of education, of which children constitute significant percentage.

Objectives: The purpose of this study was to assess the oral health status and oral hygiene habits of 6-9 years old school going children who belonged to posh and less posh areas and those who were residing in Rawalpindi but belonged to rural areas.

Study Design: The study design of the research was case control study design.

Methods: The study population consisted of 30 school going children of both sexes which were selected by convenience sampling technique. The survey was based on WHO, oral assessment form for children 2015.

Results: Evaluation of the oral health status revealed that poor oral hygiene habits were more prevalent in those schoolchildren whose parents had no or little educational background.

Conclusions: It may be concluded that at an early age, greatest need of dental health education including proper instruction of oral hygiene practices and school based preventive programs, would help children in improving preventive dental behaviour and attitude which is beneficial for their life time.
\end{abstract}

\section{INTRODUCTION}

Health is one of the major determinants that contribute to a nation's development, especially in this age of globalisation, where the health of population is considered to be the key to better productivity and efficiency of society. Oral health is essential to general health and quality of life. It is a state of being free from mouth and facial pain, oral and throat cancer, oral infections and sores, periodontal (gum) diseases, tooth decay, tooth loss, and other diseases and disorders that limit an individual's capacity in biting, chewing, smiling, speaking, and pschyco- social wellbeing (WHO).

Oral diseases are common in many societies globally, with dental caries being the most prevalent chronic disease among children. Dental caries, commonly known as tooth decay and periodontal disease affect almost $100 \%$ of population worldwide. The most common oral diseases among children are gingivitis and dental caries the later affecting $60-90 \%$ of children globally. The U.S Surgeon General report on Oral health stated that oral health problems are 5 times more likely to occur in children than asthma and 7 times more than hay fever [1].

Islamic Republic of Pakistan has population of 207,774,520, children from 0-14 years contribute almost $35.4 \%$ of the population and there is scarcity of updated data about prevalence of dental decay and other oral diseases among Pakistani school going children.

Among some few studies conducted in our region, Mohammad et al mentioned oral health challenges of Pakistan. Majority of those data which they mentioned were based on the latest and most comprehensive oral epidemiological survey of the country [2].

Reliable data on the frequency and severity of several Oral dental trauma are still lacking particularly in Pakistan. A significant portion of dental trauma relates to sports, unsafe playgrounds or schools, violence or road accidents.

The burden of Oral disease in children is significant. Most established oral disease are irreversible, and have effect on personal satisfaction and general wellbeing.

With the absence of relevant data on disparities in oral health in Pakistan, the need was felt to conduct a survey. Aim of the study was to describe, the Oral health status of school going children in Pakistan, how Oral health is linked with socio-behavioural risk factors, highlighting risk factors to Oral health in children that arise from environment, whether from school or from home, economic, social and behavioural causes and future needs for Oral health care and lastly, to encourage planners to standardize measures of Oral diseases and conditions that are important for planning and evaluation of Oral health programmes.

\section{LITERATURE REVIEW}

\section{Disparities and Poor Oral Hygiene}

Disparity means lack of equality or similarity, especially in a way that is not fair. Health disparity exists when one group receives a disproportionate share of a health burden. Differences can be most normally found along socioeconomic 
and race/ethnic line. Healthy People 2020 defines a health disparity as "a particular type of health difference that is closely linked with social, economic, and/or environmental disadvantage. Health disparities adversely affect groups of people who have systematically experienced greater obstacles to health based on their racial or ethnic group, religion, socioeconomic status, gender, age, mental health, cognitive, sensory, or physical disability, sexual orientation or gender identity, geographic location, or other characteristics historically linked to discrimination or exclusion.". Determinants of oral health disparities represent unpredictable blend of the natural, the conduct, the social, the financial, and the political.

Oral hygiene is the act of keeping one's mouth clean and free of disease and other issues (e.g. bad breath) by consistent brushing and cleaning between the teeth. It is essential that oral hygiene be carried out on a regular basis to empower counteractive actions of dental infection. Dental hygiene centres around the administration of practices important to promote oral health and prevent oral diseases.

The purpose of oral hygiene is to prevent the build-up of plaque which is the sticky film of bacteria and food that forms on the teeth. Plaque adheres to the crevices and fissures of the teeth and builds acids that, when not removed on a regular basis, slowly eat away, the protective enamel surface of the teeth, leading to cavitation. Plaque also irritates gums and can lead to gum disease, periodontal disease, and tooth loss. Brushing and flossing removes plaque from teeth, and antiseptic mouthwashes disinfect the bacteria that help in forming plaque. Fluoride, found in toothpaste, drinking water, or dental treatments, also helps to protect teeth by binding with enamel to make it stronger. In addition to such daily oral care, regular visits to the dentist promote oral health. Preventative services that the dentist can perform include fluoride treatments, sealant application, filling cavities and scaling.

The primary risk arises from a lack of proper oral hygiene practices. These major oral health problems/ risks are plaque, tartar, gingivitis, periodontitis and tooth decay (dental caries).

There have been some improvements in oral health over the past 30 years, however, inequalities still exist and are widely discussed. This is true especially for children.

Socio-economic factors are perceived as being key determinants of oral health inequalities. Inequalities in child dental health are well documented. The 2003 Children's Dental Health Survey found that children attending primary schools in socially-deprived areas of the UK were reported to have experienced more tooth decay than children in schools in non-deprived areas. Among both five and eight-year-olds, the probability of having decay into dentine or obvious decay experience of the primary teeth was about 50 per cent higher in the lowest social group than in the highest.

Disparities in dental caries prevalence associated with some ethnic minority groups are more prominent among pre-school children than in any other age group. To date, most studies have focused on the South Asian community, where religious foundation and the capacity of the mother to communicate in English have risen as imperative determinants of oral wellbeing status.

The Scottish Government action plan, Better Health, Better Care, also highlights that standards of oral health in children have a strong relationship with deprivation [3].

Hypothesis H1. There is a clear reported association between social disparity and oral hygiene for example, between dental caries and malnutrition in children. This fact is also detected in several studies.

\section{Effects of Poor Oral Hygiene on Oral Health of Children}

Oral health is multi-faceted and includes the ability to speak, smile, smell, taste, touch, chew, swallow and convey a range of emotions through facial expressions with confidence and without pain, discomfort and disease of the craniofacial complex. Oral health means more than good teeth, it is vital to general health and essential for well- being.

Child oral health is vital part of their general health, oral health is a key determinant factor for quality of life and a conceptual model by the National Committee on Vital and Health Statistics indicates that oral health outcomes of children are influenced by a triad of child level influences, including physical and demographic, development, biological and hereditary characteristics, different behaviours and practices regarding health, dental care, family level influences, socioeconomic status, social and community support, physical security, health status of parents, culture and norms and adopting abilities of the family.

Oral diseases could restrict activities in school resulting in millions of school hours to be lost each year all over the world. Moreover, the psychosocial impact of these diseases often significantly diminishes quality of life. Developing countries such as Pakistan has a high burden of common dental diseases like tooth decay and periodontal disease. Children are largely affected with dental problems due to their dietary habits. A better oral hygiene can prevent common dental and oral problems. Because of their dependence, their inability to communicate needs, young children constitute a vulnerable population. Although disparity amongst children can be found in nearly every marker of health, disparity in oral health is particularly disturbing because dental caries is the most common chronic disease of childhood [4].

Tooth extraction is a common and necessary treatment for advanced caries. Premature loss of molars is likely to result in future orthodontic problems. Therefore, children affected by ECC are likely to continue having oral health problems for which treatment is often financially out of reach for their parents specially for those who belong to lower socio-income level. 
Hypothesis H2. Literature review reveals the negative correlation between poor oral hygiene habits and good oral health. Poor oral hygiene may not only affect oral health status negatively as expressed by clinical measures but also impede and effect quality of life.

\section{Mediating Role of Poor Oral Hygiene Between Disparities and Oral Health of Pakistani Children}

Good oral health is vital to improve the general health and development specially in children of school going age. It has been reviewed that betterment in oral health may be anticipated through good understanding of an individual's knowledge and perceptions about oral health.

Worldwide, studies have highlighted differences in oral health knowledge, attitudes and practices between children and adults as well as children of different strata. Toothbrush and toothpaste are commonly used to retain good oral hygiene. However, the correct technique and frequency of tooth brushing, and concentration of fluoride in toothpaste are the laid down factors associated with prevention of dental caries. From the results of the study, it was seen that more than $80 \%$ of the children from India and Pakistan cleaned their teeth at least once a day, where majority from Pakistan (more than $60 \%$ ) practiced tooth cleaning twice a day. On the contrary, much lower tooth cleaning frequency (less than $30 \%$ ) has been reported from Indian schoolchildren by Mathur et al [5]. The practice of cleaning twice in children from Pakistan may be attributed to Muslim religion, where traditionally Muslim children are taught to use miswak at about age six that helps develop practice of brushing more often than once [6].

Oral health care is a far greater need than general healthcare, yet it is a very low priority for the majority of Pakistan people. Children grow uninformed of the importance of oral care and consequently become adults with compromised oral health, missing teeth and oral dysfunction.

Health disparity is not simply about differences between the rich and poor in society. A consistent stepwise social gradient exists for oral diseases, oral health steadily worsens in line with socioeconomic status. This social gradient is a universal phenomenon across the life course, from early childhood to older age, affecting almost all oral diseases to a varying degree, such as tooth decay, periodontal disease and oral cancers.

The interrelationship between oral and general health is proven by evidence. Despite great achievements in the oral health of populations globally, problems still remain in many communities around the world, particularly among underprivileged groups in developed and developing countries.

Hypothesis H3. Poor oral hygiene mediates the relationship between disparity and oral health of children. A recent report from one of the few longitudinal studies on oral health has indicated that the effect of poor oral health during the early years, in addition to family low socioeconomic status, is a predictor of poor oral health during adulthood [7]. Therefore, oral health inequalities in early years are likely to persist through the adult years.

Figure 1: Theoretical Framework and Hypothesis

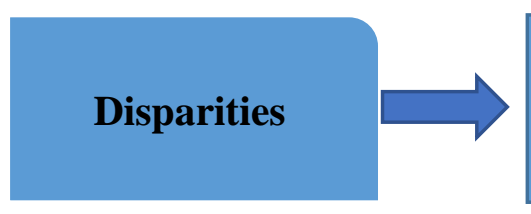

(Independent variable)

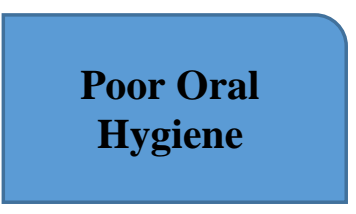

(Mediator)

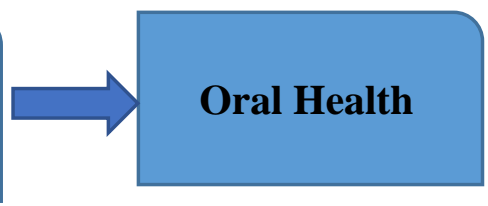

(Dependant variable)

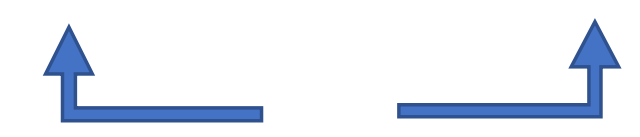

H1

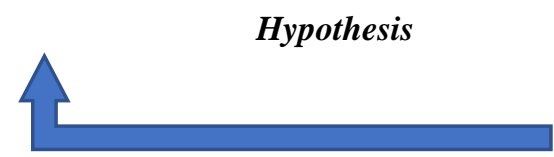

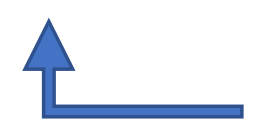

H2

Hypothesis

H3

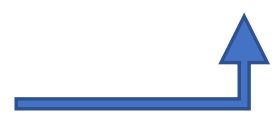

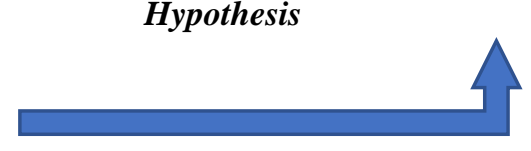

Hypothesis 


\section{Participants}

\section{METHODS}

The study was conducted to assess the disparity role on oral health condition of 6 to 9 years old school going children from Rawalpindi and children who belonged to rural areas but are residing in Rawalpindi.

Convenience sampling technique was used and 30 children were assed and examined for their oral health and oral hygiene evaluation. The respondent response rate was more than $90 \%$.

Participants consist of 30 children which were from 6 to 9 years old, among them boys were $53.3 \%$, girls were $46.7 \%$, majority of children $33.3 \%$ were of age 8 years. $80 \%$ children belonged to urban area and $20 \%$ children belonged to rural area.

\section{Instrument}

Questionnaire regarding disparity and oral hygiene of children was adopted from WHO website and oral assessment form for examination of oral health of children was also adopted from WHO basic methods-5th edition.

The instrument consists of Likert 7-point scale, with point number 4 as, not recorded option or neutral opinion was used for analysis. Reliability and Validity analysis of instruments for disparity, oral hygiene and oral health variables were conducted and recorded accordingly.

\section{Data Collection}

Data collected from different areas of Rawalpindi, including children of roots school, from streets of Chaklala, Dhol chok, children of maids originally belonging to rural area, and by online distribution of questionnaire using google docs [8].

\section{Data Analysis}

Processing and analysis of data were carried out by means of the Statistical package for the Social Sciences (SPSS - 23.0 version). From the main three variables, Frequency distribution, mean and standard deviation were calculated. Correlation analysis were performed by comparing means of disparity, oral hygiene and oral health to determine the strength of association between them.

Finally, regression analysis of variables was performed using processor by Andrew.F. Hayes, using model number 4 to access the role of oral hygiene on disparity among children.

\section{RESULTS}

30 school children, 6-9 years of age were examined, $16.7 \%$ were 6 years old, $23.3 \%$, 7 years, $33.3 \%, 8$ years and $26.7 \%$ were 9 years old. Boys, $53.3 \%$ and girls were $46.7 \%$. $80 \%$ belonged to urban and $20 \%$ to rural areas. Fathers of $10 \%$ and mothers of $26 \%$ children had no formal schooling background. $43 \%$ of children had never received dental care and the $33 \%$ who visited dentists, reason being in pain or trouble with teeth, gums or mouth. $30 \%$ of children were not satisfied with appearance of their teeth. Almost $16 \%$ kids missed their classes or school due to pain or discomfort. $23 \%$ of children used sweets and candies several times a day.

The study shows that $20 \%$ of children thought that their oral hygiene is poor while $33.3 \%$ maintained their oral hygiene well. $13 \%$ children never cleaned their teeth and the percentage of those who cleaned their teeth once a day was $40 \%$, while $10 \%$ cleaned their teeth 2 or more times a day with $83 \%$ used only toothpaste for maintaining oral hygiene. $33 \%$ knew that their tooth paste contained fluoride. Almost $83 \%$ children used toothbrush to clean their teeth along with $20 \%$ were users of dental floss. Miswak was also used by $10 \%$ of children.

Data regarding oral health condition were mainly collected by oral examination. $43 \%$ of children suffered pain or felt discomfort in teeth, and 56\% never felt pain in last 12 months. $20 \%$ children were free of caries while $20 \%$ suffered from carious teeth. In $23 \%$ children teeth were missing due to caries. Percentage of children with filled teeth with caries and without caries were $36 \%$. Gingival or gums bleeding score was positive in $56.7 \%$ children. Dental erosion was present in $13.3 \%$ and $16.7 \%$ had missing teeth due to trauma or injury. Only $10 \%$ children showed the sign of fluorosis. $33.3 \%$ children suffered from mouth ulcers.

Bivariate Correlations analysis was conducted by using computed means, of disparity variable, 4.1639, hygiene variable, 4.0694, and oral health variable, 2.5407 respectively.

The result showed highly significant negative correlation value between disparity and oral hygiene. The correlation value of hygiene and oral health showed the significant strength of association while the value between disparity and oral health variables showed significant negative relationship.

Finally, regression analysis was conducted using processor by Andrew F. Hayes, using model number 2 to analyse the total, direct and indirect effects of disparity on oral health of children. The total effect of disparity on oral health showed lower level of confidence interval and upper level of confidence interval with same negative signs LLCL $=-1.6665$ and $\mathrm{ULCL}=-.5979, \mathrm{p}=.0002$, showed the highly significant total effect negative relationship between disparity and oral health model. Direct effect showed LLCL with -1.0455 and ULCL with .4009 , meant that the result was not significant when the effect was controlled between disparity and oral health. The indirect effect of disparity and oral health when good oral 
hygiene condition was present showed the LLCL -1.5822 and ULCL $-.2984, \mathrm{p}$ value .0075 , indicating that good oral hygiene is playing significant mediating role between disparity among oral health conditions of children.

Table 1: Regression Analysis (PROCESS procedure for SPSS)

Model number 4 ( $\mathrm{Y}=$ oral health, $\mathrm{X}=$ disparity, $\mathrm{M}=$ hygiene)

Reliability Statistics

\begin{tabular}{lcc}
\hline Reliability Statistics. & Cronbach's Alpha. & N of items. \\
\hline Disparity. & 0.647. & 12 \\
Hygiene & 0.681 & 12 \\
Oral health & 0.671 & 9 \\
& &
\end{tabular}

\section{DISCUSSION}

The current study found that children with better oral hygiene habits were associated with better oral health outcome measures, while rural and no or little educational backgrounds of parents were associated with worse oral health outcome while gender had minor effect.

This study suggests that as compared to urban school-aged children, those children that belonged to rural areas and with no or little educational background of parents had increased odds of having untreated decay, oral ulcerations, dental trauma and gums decreases, caused by poor dietary habits and poor hygiene, and they have decreased odds of receiving dental care by multiple reasons. By having no dental visit, the children from very rural area are in a disadvantaged position by having less access to this preventive care. The poor oral hygiene conditions reflect the irregular tooth brushing habits of children and this study also indicates that tooth brushing is less frequent in schoolchildren belonging to less posh areas of Rawalpindi as compared with the schoolchildren of more developed and posh areas of Rawalpindi.

This study had several limitations. Firstly, this study was based on using convenience sampling technique and sample size was small which could potentially bias the results. However, the current research still bears scientific and practical value by exploring factors associated with oral health. Dental health education of children at an early age helps in improving preventive dental behaviour and attitudes, which is beneficial for a lifetime. This can be achieved by educating the parents, who are unfortunately uneducated, about dental health through school and community dental health programs.

\section{KEY POINTS}

- Children oral hygiene habits are associated with Parent's educational background.

- By having no dental visit, the children from very rural area are in a disadvantaged position by having less access to this preventive care.

- A study on oral health assessment and dental health education of children at an early age helps in improving preventive dental behaviour and attitudes, which is beneficial for a lifetime.

- For the benefit of community, a dental health programmes have to be conducted repeatedly in order to reach the goals of WHO.

- Parents should be made aware of the brushing methods, usage of preventive and importance of dental care for the children.

\section{ACKNOWLEDGMENT}

I acknowledge Professor Dr. Sajid Bashir for guiding, supporting and helping me through overall this research process. My Parents, Siblings and my Family for motivating and believing in me.

Competing interests. I, Dr. Sarah Hamid, as the only author declare that I have no competing interests in this study.

\section{REFERENCES}

[1] P. E. P. a. S. Kwan, "Evaluation of community-based oral health promotion and oral disease prevention - WHO recommendations for improved evidence in public health practice," who.int, 2004.

[2] K. AA, "Oral Health in Pakistan," researchgate.net, 2004.

[3] "Better Health, Better Care," in Action Plan The Scottish Government, 2007.

[4] "Oral health in America: A report of the Surgeon General," NIH Publication, 2000. 
[5] A. J. M. A. G. T. Mathur A, "Oral health Attitude Knowledge Behavior and Consent towards Dental treatment among School children," J Orofacial Research, 2011.

[6] K. MA, "Prevalence Dental Caries among 3-12 old children of Swat-Pakistan," Pak Oral Dent Journal, 2009.

[7] P. R. M. B. C. A. B. J. A. K. Thomson WM, "Socioeconomic inequalities in oral health in childhood and adulthood in a birth cohort.," Community Dent Oral Epidemiol, 2004.

[8] "google docs," [Online].

Available: https://docs.google.com/forms/d/1J79d0MVrdpWf5RT7QH1JJbBiwFy9NCUj7c8aXriiLI8/edit?c=0\&w=1.

[9] "Children's Dental Health Survey," in Office of National Statistics, 2003.

[10] "Dental Caries in Adolescents 12 to 19," National Institute ofDental and Craniofacial Research, 2008. 